\title{
CIÊNCIA'NATURA
}

\section{Aspectos micrometeorológicos da emissão de monoterpenos em uma floresta na Amazônia central}

\author{
Micrometeorological aspects of the monoterpenes emission in a forest at central Amazon
}

${ }^{1}$ Sarah Batalha, ${ }^{2}$ Jeong-Hoo Park, ${ }^{3}$ Eliane Alves, ${ }^{4}$ Raoni Santana, ${ }^{5}$ Roger Seco,

${ }^{6}$ Jose Oscar Vega Bustillos, ${ }^{5}$ James Smith, ${ }^{5}$ Alex Guenther e ${ }^{4}$ Julio Tóta

${ }^{1}$ Universidade Federal do Oeste do Pará/ Doutorado Sociedade, Natureza e Desenvolvimento, Brasil

${ }^{2}$ Instituto Nacional de Pesquisa Ambiental, República da Coreia

${ }^{3}$ Instituto Nacional de Pesquisas da Amazônia

${ }^{4}$ Universidade Federal do Oeste do Pará/ Instituto de Engenharia e Geociências

${ }^{5}$ Universidade da Califórnia, Irvine, Estados Unidos da América

${ }^{6}$ Instituto de Pesquisas Energéticas e Nucleares, Brasil

\begin{abstract}
Resumo
Este trabalho teve por objetivo apresentar resultados sobre a razão de mistura e fluxo de monoterpenos em uma região de floresta da Amazônia brasileira. Utilizou-se instrumentação da micrometeorologia (com o uso de um Anemômetro Sônico) e da química analítica através de um espectrômetro de massa com próton transferência (Proton Transfer Reaction - Time of Flight - Mass Spectrometer, PTR-ToF-MS). A calibração do espectrômetro foi realizada regularmente com a utilização de uma mistura gravimétrica de gás padrão contendo diferentes massas de compostos orgânicos voláteis. Os resultados evidenciaram razão de mistura média de monoterpenos de 0,185 ppbv e fluxo de emissão máxima de $1,495 \mathrm{mg} \mathrm{m}^{-2} \mathrm{~h}^{-1}$. Concluiu-se que esta região apresentou significativa emissão de monoterpenos em relação a outra floresta tropical na Amazônia central, evidenciando a necessidade de ampliar estudos sobre a química atmosférica em diferentes florestas da bacia amazônica.
\end{abstract}

Palavras-chave: Química Atmosférica. Covariância de Vórtices Turbulentos. COVBs

\section{Abstract}

This research aimed to present results about the mixing ratio and flux of monoterpenes at a forest region of the Brazilian Amazon. It was used micrometeorology instrumentation (using a Sonic Anemometer) and analytical chemistry by a Proton Transfer Reaction - Time of Flight - Mass Spectrometer, PTR-ToF-MS. The calibration of the spectrometer was performed regularly using a gravimetric mix of standard gas containing different masses of volatile organic compounds. The results showed average mixing ration of monoterpenes of $0.185 \mathrm{ppbv}$ and maximum emission flux of $1.495 \mathrm{mg} \mathrm{m}^{-2} \mathrm{~h}^{-1}$. It was concluded that this region presented a significant emission of monoterpenes in relation to another tropical forest in central Amazonia, evidencing the needing to improve studies about atmospheric chemistry in different forests of the Amazon Basin.

Keywords: Atmospheric Chemistry. Eddy Covariance. BVOCs 


\section{Introdução}

Regiões de florestas tropicais emitem naturalmente para atmosfera uma grande quantidade de compostos de carbono, os compostos orgânicos voláteis biogênicos - COVBs (GUENTHER et al. 2006). Quando liberados pela vegetação, estes compostos iniciam uma série de reações de oxidação que modificam as características químicas da troposfera, em especial da camada limite atmosférica (CLA), influenciando, assim, a formação e concentração de outros gases traços (LAOTHAWORNKITKUL et al., 2009) e aerossóis orgânicos secundários (AOSs), potenciais formadores de Núcleos de Condensação de Nuvens (NCNs) e Núcleos de Gelo (NG), que em condições de altas temperaturas, radiação solar e umidade, induzem a formação de nuvens, precipitação e deposição úmida de gases e partículas sobre florestas, como na Amazônia (KAUFMAN \& KOREN, 2006).

Embora recentes estudos na floresta tropical úmida da Amazônia brasileira tenham elucidado o grande potencial da vegetação na produção de COVBs e aerossóis orgânicos (JARDINE et al., 2015; ALVES et al., 2016; ANDREAE et al., 2017), ainda há muitas incertezas sobre os mecanismos de formação e remoção de compostos e particulados, revelando, assim, a importância de estudos sobre a química atmosférica nesta região. Além disso, forçantes de mudanças regionais e locais, tais como urbanização, diferentes formas de uso do solo, exploração madeireira e queimadas tornam o clima na Amazônia cada vez mais sensível. Em seus estudos sobre a camada limite convectiva (CLC), Fisch et al. (2004) observaram que a conversão de floresta em savana aumentou aproximadamente em 50\% a altura da CLC durante a estação seca, o que não variou na estação chuvosa ou na CLC acima da floresta em diferentes períodos. Uma das consequências a nível local para esta mudança de uso é a redução das chuvas, aumentando a quantidade de aerossol proveniente de queima de biomassa, impactando, então, a fotoquímica regional.

A determinação da emissão de gases traços, como os COVBs, é um desafio, pois para aplicação do método de medida dos vórtices turbulentos (Eddy Covariance - EC), demanda-se um equipamento que realize medidas de alta frequência (5 a $20 \mathrm{~Hz})$ e sensibilidade (BUSINGER \& DELANY, 1990). Dessa forma, este trabalho tem por objetivo apresentar parte dos resultados das medidas de razão de mistura e fluxo de monoterpenos $\left(\mathrm{C}_{10} \mathrm{H}_{16} \mathrm{H}^{+}\right)$acima do dossel de uma floresta primária, na Amazônia central, por meio da aplicação do método EC e uso de um espectrômetro de massa com próton transferência e tempo de voo da partícula ionizada (Proton Transfer Reaction - Time of Flight-Mass Spectrometer, PTR-ToF-MS).

\section{Materiais e Métodos}

A área em estudo localiza-se na Floresta Nacional (Flona) do Tapajós ( $\left.02^{\circ} 51^{\prime} \mathrm{S}, 54^{\circ} 58^{\prime} \mathrm{W}\right)$, caracterizada por ecossistema predominantemente de floresta primária tropical úmida e dossel fechado. A Flona do Tapajós foi criada pelo Decreto $\mathrm{n}^{\circ} 73.684$, em fevereiro de 1974 e devido sua biodiversidade, é considerada uma das mais importantes unidades de conservação da região norte do país, abrigando um grande volume de pesquisas científicas e didáticas no bioma Amazônia (IBAMA, 2004).

As medidas de fluxo micrometeorológico ocorreram em 16 dias do mês de junho de 2014 (período de transição entre as estações chuvosa e seca na região) e foram realizadas utilizando um Anenômetro Sônico 3D instalado próximo ao topo da torre a uma altura aproximada de 60 metros acima do nível do solo. O Anenômetro Sônico forneceu as medidas da temperatura e as componentes de velocidade do vento: zonal (u), meridional (v) e vertical (w). Os dados de $10 \mathrm{~Hz}$ foram registrados em um computador instalado no laboratório próximo à torre.

As razões de mistura dos Compostos Orgânicos Voláteis foram medidas através de um o sistema Proton Transfer Reaction - Time of Flight-Mass Spectrometer (PTR-ToF-MS), que foi desenvolvido para identificar simultaneamente um alto número de compostos orgânicos a uma resolução de tempo muito alta, superando limitações instrumentais e técnicas de metodologias anteriores (GRAUS et al., 2010).

As amostras de ar entravam por um tubo de Teflon, instalado próximo ao Sônico, a uma taxa de 40 L.min ${ }^{-1}$. Na entrada das amostras havia um filtro com suporte de PFA (Perfluoroalcóixido) e membrana de PTFE (Politetrafluoretileno), com tamanho de poro de $2 \mu \mathrm{m}$, a fim de evitar a entrada de material particulado.

O PTR-ToF-MS utilizado nesta campanha foi modelo 8000, desenvolvido pela Ionicon Analytik Gesellschaft (2008). O interior do equipamento é formado basicamente por uma parte de vácuo e outra eletrônica. No vácuo, o sistema de lentes conduz as moléculas ionizadas para o ToF-MS, enquanto este está sendo evacuado por uma bomba turbomolecular, a uma pressão cerca de $2.10^{-3}$ mbar. A parte eletrônica consiste de um microprocessador controlado através de uma tela no painel frontal do equipamento ou por um computador conectado.

Para a calibração, utilizou-se como background o ar zero produzido a partir do ar ambiente, que foi purificado passando através de um conversor catalítico (tubo de aço inoxidável preenchido com lã de vidro e revestido de platina) a $350{ }^{\circ} \mathrm{C}$; além de uma mistura gravimétrica de gás padrão contendo metanol $\left(\mathrm{CH}_{3} \mathrm{OH}\right)$, acetona $\left(\mathrm{C}_{3} \mathrm{H}_{6} \mathrm{O}\right)$, acetaldeído $\left(\mathrm{C}_{2} \mathrm{H}_{4} \mathrm{O}\right)$, isopreno $\left(\mathrm{C}_{5} \mathrm{H}_{8}\right)$, metacroleína $\left(\mathrm{C}_{4} \mathrm{H}_{6} \mathrm{O}\right)$, metil-vinil-cetona $\left(\mathrm{C}_{4} \mathrm{H}_{6} \mathrm{O}\right)$, benzeno $\left(\mathrm{C}_{6} \mathrm{H}_{6}\right)$, tolueno $\left(\mathrm{C}_{7} \mathrm{H}_{8}\right)$, $\alpha$-pineno $\left(\mathrm{C}_{10} \mathrm{H}_{16}\right), 1,2,4$-triclorobenzeno $\left(\mathrm{C}_{6} \mathrm{H}_{3} \mathrm{Cl}_{3}\right)$ e $1,3,5$-triisopropilbenzeno $\left(\mathrm{C}_{15} \mathrm{H}_{24}\right)$; padrões utilizados regularmente para proporcionar a calibração do equipamento.

Os espectros de massa foram interpretados por meio da ferramenta de processamento de dados PTRwid, programada em rotina IDL (HOLZINGER, 2015) e foram separadas as concentrações dos íons de m/z 137 e 81, que correspondem à massa de monoterpenos e fragmento de monoterpenos, respectivamente. 
Os fluxos de COVBs foram calculados usando o método da Covariância de Vórtices Turbulentos (ou Eddy Covariance), que determina o fluxo vertical dos compostos em estudo a partir do cálculo da covariância significativa entre os desvios da velocidade vertical do vento e a razão de mistura do composto orgânico identificado a cada 30 minutos (Equação 1).

Onde, $\sigma$ é a densidade do ar $\left(\mathrm{mol} \mathrm{m}^{-1}\right)$, $\mathrm{N}$ é o número de dados medidos a cada 30 minutos, ou é o desvio instantâneo da velocidade do vento vertical a partir da sua média, e ou é o desvio instantâneo da razão de mistura de isopreno a partir da sua média.

Para este cálculo, foi necessário que os dados da razão de mistura fossem medidos mais rapidamente do que o vento vertical na sua mudança de direção, por isso as medidas aplicadas ao método de vórtices turbulentos precisam ser instantâneas (STEINER \& GOLDSTEIN, 2007).

$$
F_{w c}=\frac{\sigma}{N} \sum_{i=1}^{N}\left(w_{i}-\bar{w}\right) \cdot\left(c_{i}-c\right)=\frac{\sigma}{N} \sum_{i=1}^{N} w_{i}^{\prime} \cdot c_{i}^{\prime}
$$

\section{Resultados e discussão}

Considerou-se para o cálculo do fluxo de monoterpenos $\left(\mathrm{C}_{10} \mathrm{H}_{16} \mathrm{H}^{+}\right)$as razões de mistura em ppb do fragmento principal $(81$ $\mathrm{m} / \mathrm{z}$ ) e da massa do íon principal $(137 \mathrm{~m} / \mathrm{z}$ ), conforme os critérios de análise adotados por Park et al. (2013). As razões de mistura mínima e máxima de monoterpenos foram 0,0007 e 0,547 $\mathrm{ppb}_{\mathrm{v}}$, respectivamente, com média de 0,185 ppb. Em outra floresta na Amazônia central, Yáñez-Serrano et al. (2015) observaram concentrações entre 0,23 ppb (estação chuvosa) e 0.05 ppbv (estação seca), revelando concentração duas vezes maior na Flona do Tapajós do que a média máxima observada no estudo citado. O ciclo diário de razão de mistura de monoterpenos evidenciou valores maiores nos períodos diurnos, acompanhando as médias do ciclo diário das temperaturas monitoradas no Sônico 3D (Figura 1). De acordo com Fuentes et al. (2000), os monoterpenos são geralmente independentes de luz, porém a maior emissão durante o dia é explicada pela dominância da temperatura como variável ambiental que controla a taxa de emissão.
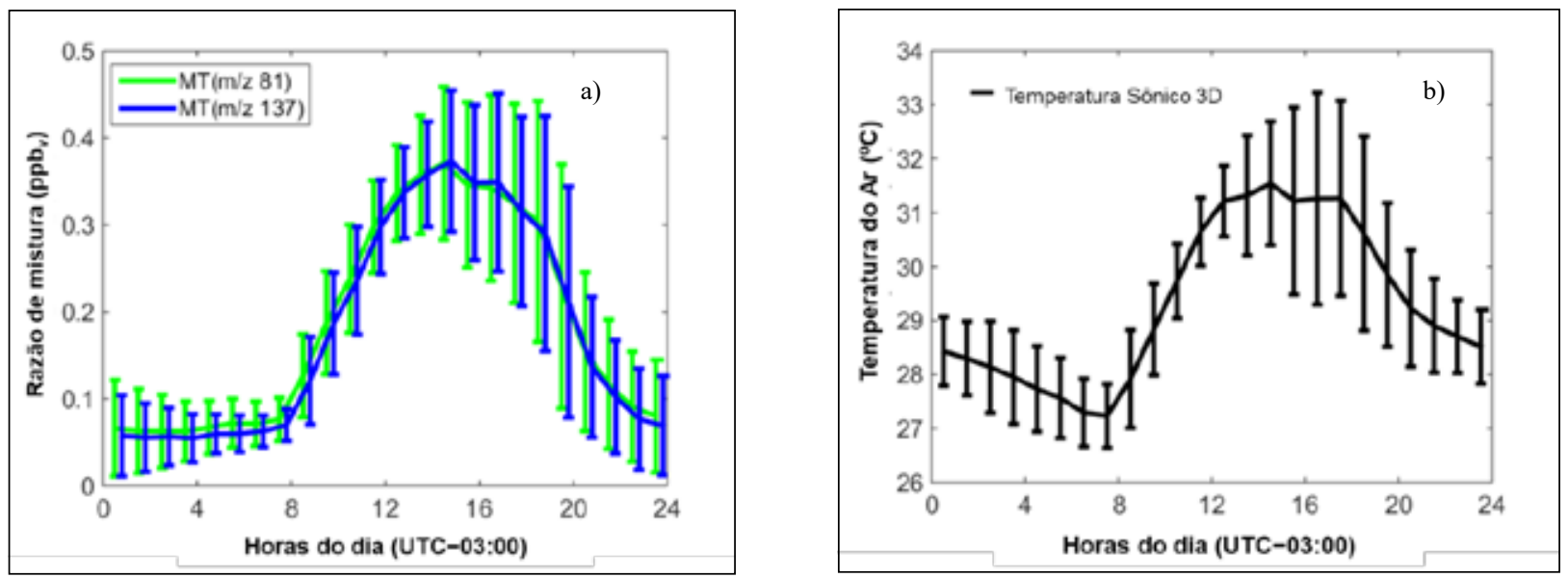

Figura 1 - (a) Médias diárias de razão de mistura de monoterpenos (MT) e (b) médias diárias de temperatura do Sônico 3D para os 16 dias de campanha.

O resultado dos cálculos de fluxos de monoterpenos foi de emissão máxima de $1,495 \mathrm{mg} \mathrm{m}^{-2} \mathrm{~h}^{-1}$, não havendo significativa variação entre os dois íons (Figura 2).

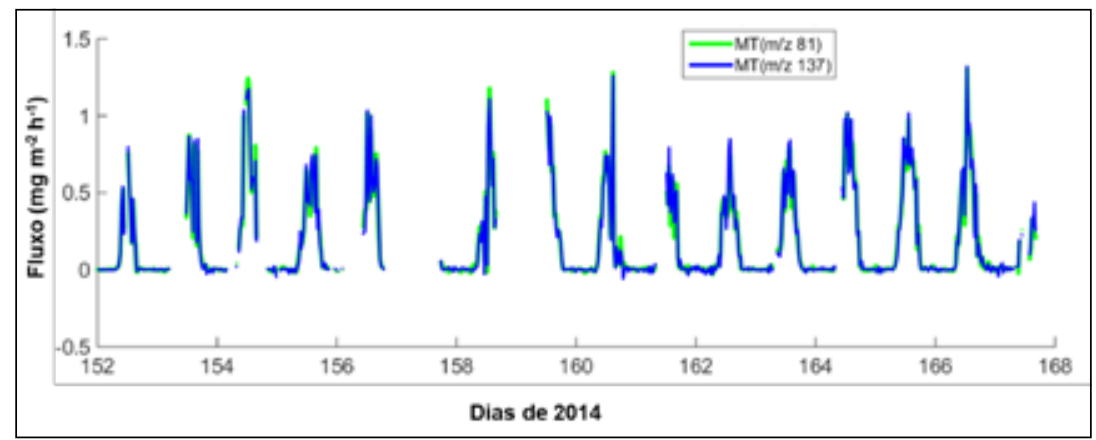

Figura 2 - Fluxo da covariância de vórtices turbulentos com base na massa dos íons identificados para os 16 dias de campanha. 


\section{Conclusão}

As informações obtidas nesta campanha compõem uma série de dados com métodos de medidas de razão de mistura e fluxo de compostos orgânicos voláteis realizadas pela primeira vez nesta região de floresta tropical. Os resultados evidenciaram a necessidade em se aprofundar estudos sobre química atmosférica e a micrometeorologia em diferentes regiões do bioma Amazônia, visto que diferenças sensíveis na concentração e fluxo dos COVBs podem revelar as variações das emissões em diversos ambientes que compõe o grande mosaico de florestas da enorme bacia amazônica. Espera-se que esta pesquisa contribua para reforçar a importância da floresta no controle climático local e regional, além de contribuir para o melhoramento de modelos de estimativas globais de emissão de COVBs.

\section{Agradecimentos}

Os autores expressam seus agradecimentos à Coordenação de Aperfeiçoamento de Pessoal de Nível Superior (CAPES) pela bolsa de estágio sanduíche da primeira autora; à Fundação Amazônia de Amparo a Estudos e Pesquisas do Pará (FAPESPA) pela bolsa de doutorado da primeira autora; à Universidade Federal do Oeste do Pará (UFOPA); à Universidade da Califórnia, Irvine (UCI); ao Instituto Nacional de Pesquisas da Amazônia (INPA) em Santarém-Pa e ao Instituto Chico Mendes de Conservação da Biodiversidade (ICMBio).

\section{Referências}

ALVES, E. G., JARDINE, K., TOTA, J., JARDINE, A., YÃNEZ-SERRANO, A. M., KARL, T., TAVARES, J., NELSON, B., GU, D., STAVRAKOU, T., MARTIN, S., ARTAXO, P., MANZI, A., AND GUENTHER, A.:

Seasonality of isoprenoid emissions from a primary rainforest in central Amazonia. Atmospheric Chemistry and Physics. 2016; 16: 3903-3925.

ANDREAE, M. O., AFCHINE, A., ALBRECHT, R., HOLANDA, B. A., ARTAXO, P., BARBOSA, H. M. J., BORMANN, S., CECCHINI, M. A., COSTA, A., DOLLNER, M., FÜTTERER, D., JÄRVINEN, E., JURKAT, T., KLIMACH, T., KONEMANN, T., KNOTE, C., KRÄMER, M., KRISNA, T., MACHADO, L. A. T., MERTES, S., MINIKIN, A., PÖHLKER, C., PÖHLKER, M. L., PÖSCHL, U., ROSENFELD, D., SAUER, D., SCHLAGER, H., SCHNAITER, M., SCHNEIDER, J., SCHULZ, C., SPANU, A., SPERLING, V. B., VOIGT, C., WALSER, A., WANG, J., WEINZIERL, B., WENDISCH, M., AND ZIEREIS, H.. Aerosol characteristics and particle production in the upper troposphere over the Amazon Basin, Atmospheric Chemistry and Physics, in review, 2017.

BUSINGER, J. A.; DELANY, A. C.: Chemical Sensor Resolution Required for Measuring Surface Fluxes by 3 Common Micrometeorological Techniques, Journal of Atmospheric Chemistry. 1990; 10: 399-410.

FISCH, G., TOTA, J., MACHADO, L. A. T., DIAS, M., LYRA, R. F. D., NOBRE, C. A., DOLMAN, A. J., GASH, J. H. C.. The convective boundary layer over pasture and forest in Amazonia. Theoretical and Applied Climatology. 2004; 78: 47 - 59 .

FUENTES, J. D.; LERDAU, M.; ATKINSON, R.; BALDOCCHI, D.; BOTTENHEIM, J. W.; CICCIOLI, P.; LAMB, B.; GERON, C.; GU, L.; GUENTHER, A.; SHARKEY, T. D.; STOCKWELL, W.. Biogenic hydrocarbons in the atmospheric boundary layer: A review. Bulletin of the American Meteorological Society. $2000 ; 81$ (7): $1537-1575$.

GUENTHER, A., KARL, T., HARLEY, P., WIEDINMYER, C., PALMER, P. I., GERON, C.: Estimates of global terrestrial isoprene emissions using MEGAN (Model of Emissions of Gases and Aerosols from Nature), Atmospheric Chemistry and Physics. 2006; 6: 3181-3210.

GRAUS, M., MULLER, M., HANSEL, A.: High Resolution PTRTOF: Quantification and Formula Confirmation of VOC in Real Time, Journal of the American Society for Mass Spectrometry. 2010; 21: 1037-1044.

HOLZINGER, R.. PTRwid: A new widget tool for processing PTR-TOF-MS data. Atmospheric Measurement Techniques. 2015; 8: 3903-3922.

IBAMA. Plano de Manejo da Floresta Nacional de Tapajós. Brasília: IBAMA, 2004. 
JARDINE, A. B. et al. Highly reactive light-dependent monoterpenes in the Amazon. Geophysical Research Letters. 2015; 42: 1576-1583.

KAUFMAN, Y. J., KOREN, I. Smoke and pollution aerosol effect on cloud cover. Science. 2006; 313, 655-658.

LAOTHAWORNKITKUL, J., TAYLOR, J. E., PAUL, N. D., HEWITT, C. N.. Biogenic volatile organic compounds in the Earth system. New Phytologist, 2009; 183: 27-51.

PARK, J.-H., GOLDSTEIN, A. H., TIMKOVSKY, J., FARES, S., WEBER, R., KARLIK, J., HOLZINGER, R.: Eddy covariance emission and deposition flux measurements using proton transfer reaction - time of flight - mass spectrometry (PTR-TOF-MS): comparison with PTR-MS measured vertical gradients and fluxes, Atmospheric Chemistry and Physics. 2013; 13: 1439-1456.

STEINER, A. H.; GOLDSTEIN, A. L.. Biogenic VOCs. In: KOPPMANN, R., 2007. Volatile organic compounds in the atmosphere. $1^{\mathrm{a}}$ ed. Orxford: Blackwell, cap 3, pp 82-128.

YÁÑEZ-SERRANO, A. M., NÖLSCHER, A. C., WILLIAMS, J., WOLFF, S., ALVES, E., MARTINS, G. A., BOURTSOUKIDIS, E., BRITO, J., JARDINE, K., ARTAXO, P., KESSELMEIER, J.: Diel and seasonal changes of biogenic volatile organic compounds within and above an Amazonian rainforest, Atmospheric Chemistry and Physics. 2015; 15, 3359-3378.

Sarah Batalha

Universidade Federal do Oeste do Pará/ Doutorado Sociedade, Natureza e Desenvolvimento, Brasil E-mail: ssa.batalha@gmail.com

Jeong-Hoo Park

Instituto Nacional de Pesquisa Ambiental, República da Coreia E-mail: jeonghoo@korea.kr

Eliane Alves

Instituto Nacional de Pesquisas da Amazônia E-mail: elianegomes.alves@gmail.com

\section{Raoni Santana}

Universidade Federal do Oeste do Pará/ Instituto de Engenharia e Geociências E-mail: raoniass@gmail.com

Roger Seco

Universidade da Califórnia, Irvine, Estados Unidos da América E-mail: rseco@uci.edu

Jose Oscar Vega Bustillos

Instituto de Pesquisas Energéticas e Nucleares, Brasil E-mail: ovega@ipen.br

James Smith

Universidade da Califórnia, Irvine, Estados Unidos da América E-mail: jimsmith@uci.edu

Alex Guenther

Universidade da Califórnia, Irvine, Estados Unidos da América E-mail: alex.guenther@uci.edu

Julio Tóta

Universidade Federal do Oeste do Pará/ Instituto de Engenharia e Geociências E-mail: totaju@gmail.com 